\title{
Electronic structure and luminescence of $\mathrm{CsI}: \mathrm{Na}$
}

\author{
C K Ong†, K S Songł, R Monnier§ and A M Stoneham \\ Theoretical Physics Division, AERE Harwell, Didcot, Oxon, UK
}

Received 13 February 1979

\begin{abstract}
Calculations are performed on several aspects of the luminescence of pure CsI and CsI: Na. These include electronic-structure calculations by both pseudopotential and semiempirical molecular-orbital methods, as well as lattice-configuration studies. The results suggest that the main observed emission in CsI : Na at $2.95 \mathrm{eV}$ involves the recombination of a self-trapped exciton immediately adjacent to the substitutional $\mathrm{Na}$ impurity.
\end{abstract}

\section{Introduction}

Sodium-doped CsI is a remarkably effective $\mathrm{x}$-ray phosphor. The high atomic numbers of Cs and I give strong $\mathrm{x}$-ray absorption, and there is an efficient blue luminescence associated with the sodium ions. The CsI: $\mathrm{Na}$ system is also an example of ionic systems which are discussed intermittently in the literature and which parallel the isoelectronic (or isovalent) systems much studied in semiconductors.

Even though CsI : Na has been developed to the point of practical application (Stevels 1975, Stevels and Pingault 1975), there is much uncertainty about the precise mechanisms which lead to the blue luminescence. One complication has been doubt about which of the observed luminescence bands are intrinsic and which are sodium-associated. Another uncertainty concerns the electronic and ionic states involved; this has been the subject of both vague and unlikely suggestions. In this note we shall bring together a number of separate calculations which relate to CsI: $\mathrm{Na}$ and which, collectively, limit the number of possible interpretations. Various authors have suggested that iodine vacancies (Stevels 1976), F-centres (Salau 1978) or sodium interstitial atoms (Basil'chuk et al 1977) are involved with the sodium. We shall not discuss these possibilities here, except to note that substitutional sodium is probably the dominant imperfection.

\section{Charge states of $\mathrm{Na}$ in $\mathrm{CsI}$}

Substitutional $\mathrm{Na}$ in CsI can exist in at least three charge states. The simplest is just $\mathrm{Na}^{+}$ replacing $\mathrm{Cs}^{+}$. This small substitutional ion will obviously distort the surrounding lattice. It is likely but not certain that the displacement field is principally an inward radial distortion of the neighbours, rather than the off-centre behaviour of the kind

\footnotetext{
$\uparrow$ Permanent address: Physics Department, Ahmadu-Bello University, Zaria, Nigeria.

¥ Permanent address: Physics Department, University of Ottawa, Ottawa, Ontario, Canada.

$\S$ Permanent address: Laboratorium für Festkörperphysik, CH-8049 Zürich, Hönggerberg, Switzerland.
} 
seen in $\mathrm{KCl}: \mathrm{Li}$. The second charge state involves the capture of an electron by the sodium ion to give $\left[\mathrm{Na}^{+} \mathrm{e}\right]$. The binding results from the greater electron affinity of $\mathrm{Na}^{+}$, modified by the altered lattice distortion. Analogous charge states have been noted by Park and Faust (1966) for $\mathrm{KI}: \mathrm{Na}$ and by Schneider (1978) for $\mathrm{KCl}: \mathrm{Na}$; the $\mathrm{KCl}: \mathrm{Br}$ system (Hinks and Susman 1977) may also be analogous. The third charge state involves the capture of a hole. In alkali halides, the hole is self-trapped (the $\mathrm{V}_{\mathrm{k}}$ centre). The binding of sodium and hole is principally elastic, and the system is best described as $\left[\mathrm{Na}^{+} \mathrm{V}_{\mathrm{k}}\right]$. We shall now describe some calculations on $\left[\mathrm{Na}^{+} \mathrm{e}\right]$ and $\left[\mathrm{Na}^{+} \mathrm{V}_{\mathrm{k}}\right]$.

\subsection{The $\left[\mathrm{Na}^{+} e\right]$ system}

Calculations of the substitutional sodium ion as an electron trap have already been reported by Monnier (1976). He concluded that the electron was bound by $0.45 \mathrm{eV}$, and that photoionisation $\left(\left[\mathrm{Na}^{+} \mathrm{e}\right] \rightarrow\left[\mathrm{Na}^{+}\right]+\mathrm{e}\right)$ was the origin of the observed peak at $1.72 \mathrm{eV}$. The final state is a p-like resonance in the continuum of the conduction band states, into which it can decay rapidly. The transition thus parallels that giving the D line in the free atom. The peak lies higher than the photoionisation threshold because of the form of the density of states of the conduction band. Monnier also proposed that it is a relaxed excited state of $\left[\mathrm{Na}^{+} \mathrm{e}\right]$ which has binding corresponding to the observed $1.6 \mathrm{meV}$ thermal ionisation energy. This weak binding is caused primarily by the distortion field.

We have made additional calculations which parallel Monnier's and which support his conclusions about the ground state. The present calculations have used the point-ion model with ion-size corrections (Bartram et al 1968). The most important conclusions are that the general magnitude of the binding is rather less than $1 \mathrm{eV}$, in broad agreement with Monnier, and that the wavefunctions contain a very extended component, with effective radii of $4-6 \AA$. The method used is not especially accurate for such extended states, principally because of limitations involved in handling the distortion field. The calculations suggest that a p-like bound state lies very close to the $1 \mathrm{~s}$-like ground state. There is no significant change in the binding if the impurity is moved a small distance from the precise substitutional site.

\subsection{The $\left[\mathrm{Na}^{+} \mathrm{V}_{k}\right]$ system}

The self-trapped holes in the caesium halides have been discussed by Monnier et al (1977). The present work extends their treatment to cover two configurations of $V_{k}$ centres near $\mathrm{Na}^{+}$substitutional ions. Like the earlier work, the calculations use the HADES program (Lidiard and Norgett 1972), and we have adopted the same interatomic potentials.

The $\mathrm{V}_{\mathrm{k}}$ centre in CsI can be regarded as an $\mathrm{I}_{2}^{-}$molecular ion replacing two adjacent $\mathrm{I}^{-}$ions. The two configurations we have considered are (i) configuration $\mathrm{A}$, in which the two $\mathrm{I}^{-}$ions replaced by $\mathrm{I}_{2}^{-}$are both nearest neighbours to the $\mathrm{Na}^{+}$, and (ii) configuration $\mathrm{B}$, in which only one of these $\mathrm{I}^{-}$is a nearest neighbour. The most important result is that the closer arrangement $\mathrm{A}$ is lower in energy by about $0.3 \mathrm{eV}$. The precise figure depends on the assumed weak $\mathrm{Cs}-\mathrm{Na}$ attractive interaction. If it is taken to be the same as the Cs-Cs interaction, $E_{\mathrm{A}}$ is lower than $E_{\mathrm{B}}$ by $0.33 \mathrm{eV}$; if the $\mathrm{Cs}-\mathrm{Na}$ interaction is reduced by a factor of ten, the difference is $0.35 \mathrm{eV}$. Both these figures suggest an interaction which is primarily elastic, and a thermal binding energy $\left(\left[\mathrm{Na}^{+} \mathrm{V}_{k}\right] \rightarrow\left[\mathrm{Na}^{+}\right]+\mathrm{V}_{\mathrm{k}}\right)$ of around $0.4 \mathrm{eV}$. 


\section{Luminescence and the exciton}

Recombination luminescence has been observed by both optical and $x$-ray excitation of CsI: Na. In optical absorption, where the band gap is $6.3 \mathrm{eV}$, the intrinsic exciton is at $5.81 \mathrm{eV}$, and Na-associated bands appear at 5.44 and $5.69 \mathrm{eV}$. When there is very little Na present, a self-trapped exciton might be expected to form (in essence $\left[\mathrm{V}_{\mathrm{k}} \mathrm{e}\right]$ ), showing the characteristic radiative and non-radiative recombination. When $x$-ray excitation occurs, we presume that a core hole is formed which later changes into a self-trapped hole and expect more $V_{k}$ centres separated from their electrons than when optical excitation is used.

The blue luminescence of CsI: $\mathrm{Na}$ comes primarily from a $2.95 \mathrm{eV}$ band. Several different recombination mechanisms are possible, including these associated with $\mathrm{Na}^{+}$:

(i) long-distance tunnelling recombination, as for donor-acceptor pairs in semiconductors,

$$
\left[\mathrm{Na}^{+} \mathrm{e}\right]+\mathrm{V}_{\mathrm{k}} \rightarrow\left[\mathrm{Na}^{+}\right]+h v ;
$$

(ii) long-distance electron transfer to form a self-trapped exciton intermediate,

$$
\left[\mathrm{Na}^{+} \mathrm{e}\right]+\mathrm{V}_{\mathrm{k}} \rightarrow\left[\mathrm{Na}^{+}\right]+\left[\mathrm{V}_{\mathrm{k}} \mathrm{e}\right] \rightarrow\left[\mathrm{Na}^{+}\right]+h v ;
$$

(iii) capture to form a sodium-localised, self-trapped exciton,

$$
\left[\mathrm{Na}^{+} \mathrm{V}_{\mathrm{k}}\right]+\mathrm{e} \rightarrow\left[\mathrm{Na}^{+} \mathrm{V}_{\mathrm{k}} \mathrm{e}\right] \rightarrow\left[\mathrm{Na}^{+}\right]+h v ;
$$

(iv) recombinative capture of a free electron

$$
\left[\mathrm{Na}^{+} \mathrm{V}_{\mathrm{k}}\right]+\mathrm{e} \rightarrow\left[\mathrm{Na}^{+}\right]+h \nu .
$$

There are many other possibilities with different degrees of complexity, but these four contain most of the important elements. One important distinction among these options is whether recombination is direct although modified by the $\mathrm{Na}^{+}$(mechanisms $i$ and iv) or whether an intermediate species is formed, like the self-trapped exciton $\left[\mathrm{V}_{\mathrm{k}} \mathrm{e}\right]$ in (ii) or its trapped analogue in (iii). There is strong experimental evidence for mechanisms like (ii) or (iii). Pellaux et al (1978) have shown that, if aligned $V_{k}$ centres are used, luminescence is polarised with respect to the $V_{k}$ centre axis, rather than related to the random directions of nearby $\mathrm{Na}^{+}$ions. Bates et al $(1977)$ found no polarised luminescence after excitation by polarised band-gap light. This does not rule out the self-trapped species, as they suggest. Probably their null result comes simply from the selftrapped excitons produced by the exciting light and from the reorientation which can occur in the excited states of self-trapped excitons. The doubts in the experimental literature about whether certain bands are intrinsic or Na-induced also support models like (ii) and (iii), since the differences seen would be kinetic (i.e. in the route to the luminescent state) rather than distinct final transitions. Our later results support model (iii).

\subsection{Electronic structure of the self-trapped exciton in CsI}

This system parallels the self-trapped excitons in the NaCl-structure alkali halides in many respects. It can be considered as an electron trapped by a self-trapped hole, $\left[\mathrm{V}_{\mathrm{k}} \mathrm{e}\right]$. The main features of the lowest state, both in electronic structure (Fowler et al 1973) and in the kinetics of transitions amongst the low-lying states, closely resemble other self-trapped excitons. The main differences stem from the different geometry of the host 
Table 1. Comparison of the recombination and electron-excited energies (in eV) of the selftrapped exciton in CsI. The $0.60 \mathrm{eV}$ experimental value is the difference between the 4.27 and $3.67 \mathrm{eV}$ bands and is queried because the precise states are in doubt.

\begin{tabular}{llll}
\hline & Pseudopotential & CXDO experimental \\
\hline Recombination energy & & & \\
from lowest state $\left(\mathrm{A}_{1 \mathrm{~g}}\right)$ & & $3 \cdot 11$ & 3.67 \\
$\mathrm{~A}_{1 \mathrm{~g}}-\mathrm{B}_{\mathrm{u}}$ separation & 0.66 & 0.97 & \\
$\mathrm{~A}_{1 \mathrm{~g}}-\mathrm{E}_{\mathrm{u}}$ separation & 0.34 & 1.30 & $0.60 ?$ \\
$\mathrm{~A}_{1 \mathrm{~g}}-\mathrm{A}_{1 \mathrm{~g}}$ separation & 1.11 & 1.38 & \\
Ionisation energy & & $(4.02)$ & \\
\hline
\end{tabular}

lattice and from the large spin-orbit coupling. In CsI, whilst selection rules obviously do exist (T Iida, private communication 1978), the distinction between spin-allowed and spin-forbidden optical transitions is inappropriate.

Pellaux et al (1978) discuss two principal emissions from self-trapped exciton recombination: one at $4 \cdot 27 \mathrm{eV}$, with both $\pi$ and $\sigma$ polarised components, and one at $3.67 \mathrm{eV}$, which is purely $\pi$ polarised. Other workers note 'intrinsic' bands at 4.0 and $2.95 \mathrm{eV}$. We have made two sets of calculations which are related to these results. In the first, the point-ion model with ion-size corrections was used to deduce the energy levels of the electron in the potential of the $\mathrm{V}_{\mathrm{k}}$ centre. This method is like that used by Song et al (1975) and uses the PRISM code. The levels are listed under 'Pseudopotential' in

Table 2. CNDo parameters

\begin{tabular}{llllllll}
\hline & \multicolumn{3}{c}{ Orbital experiments (au) } & \multicolumn{3}{c}{ Ionisation energies (eV) } & \multicolumn{3}{c}{ Bonding parameters (eV) } \\
Ion & $\zeta_{\mathrm{sp}}$ & $\zeta_{\mathrm{d}}$ & $I_{\mathrm{s}}$ & $I_{\mathrm{p}}$ & $I_{\mathrm{d}}$ & $\beta_{\mathrm{sp}}$ & $\beta_{\mathrm{d}}$ \\
\hline $\mathrm{Cs}^{+}$ & 1.35 & 5.71 & 0.72 & 0.45 & 0.1 & -4.70 & -4.6 \\
$\mathrm{I}^{-}$ & 2.15 & 5.23 & 18.0 & 5.06 & 0.2 & -6.65 & -3.0 \\
\hline
\end{tabular}

table 1 . In the second approach, semiempirical molecular-orbital calculations were performed using the CNDO method embodied in the Harwell MOSES code. The method is like that used by Itoh et al (1975), and uses CNDO parameters derived by Ong (1977). The parameters are listed in table 2. In table 3 the various properties of CsI molecules and perfect crystals are predicted using the same parameters, and confirm agreement with experiment. It should be stressed, however, that these parameters do not represent the best that might be achieved ultimately, though they should be a satisfactory working

Table 3. C.NDO Predictions for the CsI molecule and perfect solid

\begin{tabular}{llll}
\hline CsI molecule & Bond length $(\AA)$ & Dissociation energy (eV) & Dipole moment (Debye) \\
\hline Theory & 3.32 & 3.57 & $11 \cdot 18$ \\
Experiment & 3.32 & 3.57 & $12 \cdot 1$ \\
\hline Crystal & Valence band width $(\mathrm{eV})$ & Band gap (eV) & Comment \\
\hline Theory & 3.29 & 5.50 & 40 atom cluster plus \\
Experiment & 3.2 & 6.2 & surrounding point ions \\
\hline
\end{tabular}


set. The discrepancies between the methods may result partly from the limitations of the parameters. There will also be a contribution from the difference in the precise lattice geometries used. Nevertheless, there is an encouraging degree of consistency, and broad agreement with available data.

\subsection{Effects of $\mathrm{Na}$ on the self-trapped exciton in CsI}

The $\mathrm{Na}$ impurity has several possible effects on the self-trapped exciton, caused by both its higher electronegativity and its distortion of the lattice. In this section we discuss these effects in relation to the strong emissions seen at $3.67 \mathrm{eV}$ in pure CsI and at $2.95 \mathrm{eV}$ in Na-doped CsI. In particular, we verify that model (iii) of $\S 3.1$ fits the observed spectra.

The easiest way to relate the recombination energy for the process involving the defect-localised, self-trapped exciton $\left[\mathrm{Na}^{+} \mathrm{V}_{\mathrm{k}} \mathrm{e}\right] \rightarrow\left[\mathrm{Na}^{+}\right]+\hbar \omega_{\mathrm{Na}}$ to the intrinsic process $\left[\mathrm{V}_{\mathrm{k}} \mathrm{e}\right] \rightarrow \hbar \omega_{0}$ is to consider an (imaginary) energy cycle in which first the electron and then the $\mathrm{V}_{\mathrm{k}}$ centre are removed from the $\mathrm{Na}^{+}$ion. This shows that we can write $\hbar \omega_{\mathrm{Na}}=\hbar \omega_{0}-E_{\mathrm{v}}-\left(E_{\mathrm{I}}-E_{0}\right)$ where $E_{\mathrm{v}}$ is the binding of the $\mathrm{V}_{\mathrm{k}}$ centre to the $\left[\mathrm{Na}^{+}\right]$, and where $E_{I}$ and $E_{0}$ are the energies needed to remove the electron from $\left[\mathrm{Na}^{+} \mathrm{V}_{\mathrm{k}} \mathrm{e}\right]$ and $\left[\mathrm{V}_{\mathrm{k}} \mathrm{e}\right]$ respectively.

The energy $E_{\mathrm{v}}$ was estimated as $0.4 \mathrm{eV}$ in $\S 2.2$. The difference $\left(E_{\mathrm{I}}-E_{0}\right)$ is also small, and it cannot be estimated very accurately by the full variational pseudopotential method. However, we can use the pseudopotential wavefunctions and Hamiltonian to obtain a prediction from first-order perturbation theory. Thus the expectation value of the difference in the $\mathrm{Cs}^{+}$and $\mathrm{Na}^{+}$ion-size corrections can be calculated using the unperturbed wavefunction. We have therefore included only the effect of the altered electronegativity of the $\mathrm{Na}^{+}$relative to $\mathrm{Cs}^{+}$. There is an implicit assumption (consistent with almost all earlier work on the self-trapped exciton) that the trapped electron is sufficiently diffuse to not alter the local geometry significantly. The estimate gives $E_{\mathrm{I}}-E_{0} \simeq 0.3 \mathrm{eV}$. Thus we predict that recombination of a self-trapped exciton adjacent to $\left[\mathrm{Na}^{+}\right]$will occur at $E_{\mathrm{v}}+\left(E_{\mathrm{I}}-E_{0}\right) \simeq 0.7 \mathrm{eV}$ lower energy than the recombination of an isolated self-trapped exciton. Since the observed difference between the strong extrinsic and intrinsic bands is $3.67-2.95 \simeq 0.72 \mathrm{eV}$ and in the right direction, we make these tentative assignments: (i) $3.67 \mathrm{eV}$ band-intrinsic self-trapped exciton recombination; (ii) $2.95 \mathrm{eV}$ band-recombination of self-trapped exciton adjacent to $\mathrm{Na}^{+}$impurity. We note that the $\left[\mathrm{Na}^{+} \mathrm{V}_{\mathrm{k}} \mathrm{e}\right]$ system can be aligned in the same way as $\left[\mathrm{V}_{\mathrm{k}} \mathrm{e}\right]$ and that it should show similar dichroism.

A problem emerges when these results are compared with those for $\mathrm{KCl}: \mathrm{Na}$ (Hirano and Itoh 1977) and $\mathrm{KBr}: \mathrm{Na}$ (Toyoda et al 1976) where a blue shift is observed, rather than the red shift expected from arguments like those already presented. Clearly the different crystal structures may have some effect. It seems more likely, however, that the intrinsic and extrinsic transitions seen in $\mathrm{KCl}: \mathrm{Na}$ and $\mathrm{KBr}: \mathrm{Na}$ do not correspond precisely. Whereas the observed transition is merely shifted by $\mathrm{Na}$ in CsI, quite different electronic states appear to be involved in $\mathrm{KBr}$ and $\mathrm{KCl}$, depending on whether $\mathrm{Na}$ is present or not.

\section{References}

Bartram R H, Stoneham A M and Gash P 1968 Phys. Rev. 1761014

Basil'chuk V G, Panova A N and Shakhova K V 1977 Sov. Phys.-Solid. St. 19686

Bates C W, Salau A and Leniart D 1977 Phys. Rel: B 155963 
Fowler W B, Marrone M J and Kabler M N 1973 Phys. Rev. B 85909

Hinks D and Susman S 1977 Phys. Stat. Solidi b 52 K53

Hirano Y and Itoh N 1977 Phys. Lett. 60A 465

Hsu O and Bates C W 1977 Phys. Rev. B 155821

Itoh N, Stoneham A M and Harker A H 1977 J. Phys. C: Solid St. Phys. C 1041.97

Lidiard A B and Norgett M J 1972 Computational Solid State Physics (ed F Herman, N W Dalton and T R Koehler (New York: Plenum) p 385

Monnier R 1976 Solid St. Commun. 19681

Monnier R, Song K S and Stoneham A M 1977 J. Phys. C: Solid St. Phys. 104441

Ong K 1977 to be published

Park K and Faust W L 1966 Phys. Rev. Lett. 17137

Pellaux J P, Iida T and Aegerter M A 1979 Gatlinburg Color Center Conference Abstracts p 336

Poole T R, Jenkin J G, Liesegang J and Leckey R C G 1975a Phys. Rev. B 115179 1975b Phys. Rev. B 115190

Salau A M 1978 Phys. Lett. 67 A 157

Schneider I 1978 Solid St. Commun. 251027

Sidler T 1976 Thesis University of Neuchâtel

Song K S, Stoneham A M and Harker A H 1975 J. Phys. C: Solid St. Phys. 81125

Stevels A L N and Pingault F 1975 Philips Res. Rep. 30277

Stevels A L N 1975 Medicamundi 2012 1976 J. Lumin. 12/1397

Stoneham A M 1975 Theory of Defects in Solids (Oxford: Oxford University Press)

Thiebaud O, Pilloud J J, Aegerter M A and Jaccard C 1976 J. Phy sique. 37 C7-169

Toyoda K, Nakamura K and Nakai Y 1976 J. Phys. Soc. Japan 411981 